\title{
Assessment of the European classification criteria for Sjögren's syndrome in a series of clinically defined cases: results of a prospective multicentre study
}

Claudio Vitali, Stefano Bombardieri, Haralampos M Moutsopoulos, Joaquin Coll, Roberto Gerli, Pierre Y Hatron, Louis Kater, Yriö T Konttinen, Rolf Manthorpe, Olivier Meyer, Marta Mosca, Pierantonio Ostuni, Raffaele A Pellerito, Yvon Pennec, Stephen R Porter, Andrea Richards, Bernard Sauvezie, Morten Schiødt, Maria Sciuto, Yehuda Shoenfeld, Fotini N Skopouli, Josef S Smolen, Francisco Soromenho, Moshe Tishler, Matija Tomsic, Joop P van de Merwe, Christine M Yeoman, Marie J Wattiaux (The European Study Group on Diagnostic Criteria for Sjögren's Syndrome ${ }^{\star}$ )

\begin{abstract}
Objective-To assess the recently proposed preliminary criteria for the classification of Sjögren's syndrome (SS) in a multicentre European study of a new series of clinically defined cases.

Methods-The criteria included six items: I = ocular symptoms; II = oral symptoms; III = evidence of keratoconjunctivitis sicca; IV = focal sialoadenitis by minor salivary gland biopsy; $V=$ instrumental evidence of salivary gland involvement; VI = presence of autoantibodies. Each centre was asked to provide five patients with primary SS, five with secondary SS, five with connective tissue diseases (CTD) but without SS, and five controls (patients with ocular or oral features that may simulate SS). The preliminary six item classification criteria set was applied to both the SS patients and the non-SS controls, and the performance of the criteria in terms of sensitivity and specificity was tested.

Results-The criteria set was tested on a total of 278 cases (157 SS patients and 121 non-SS controls) collected from 16 centres in 10 countries. At least four of the six items in the criteria set (limiting item VI to the presence of $\mathrm{Ro}(\mathrm{SS}-\mathrm{A})$ or $\mathrm{La}(\mathrm{SS}-\mathrm{B})$ antibodies) were present in 79 of 81 patients initially classified as having primary SS (sensitivity $97 \cdot 5 \%$ ), but in only seven of 121 non-SS controls (specificity $94 \cdot 2 \%$ ). When the presence of item I or II plus any two of items III-V of the criteria set was considered as indicative of secondary SS, $97.3 \%$ (71 of 73) of the patients initially defined as having this disorder and $91.8 \%$ (45 of 49) of the control patients with CTD without SS were correctly classified.

Conclusion-This prospective study confirmed the high validity and reliability of the classification criteria for SS recently proposed by the European Community Study Group.
\end{abstract}

(Ann Rheum Dis 1996; 55: 116-121)
Because most rheumatic diseases lack a single distinguishing feature, every disease is usually identified by the presence of a combination of clinical and laboratory manifestations. Classification criteria for most of the rheumatic disorders have been proposed and validated ${ }^{1-6}$ to establish the combination of disease features most useful for the diagnostic approach, to provide a uniform language, and to facilitate the comparison of different patient populations between studies. ${ }^{1}$

Recently, a group of investigators sponsored by the European Community defined a preliminary criteria set for the classification of Sjögren's syndrome (SS), ${ }^{7}$ a disease for which different sets of criteria have in the past been proposed by different groups, but none of which has received universal acceptance. ${ }^{8-12}$

In the present work, the accuracy of the European preliminary criteria in correctly classifying SS patients and disease controls was assessed. This study was carried out by 16 different centres throughout Europe, some of which joined the study at this point (that is, they did not take part in the previous study on the definition of classification criteria).

\section{Patients and methods}

STUDY PROTOCOL

The study protocol was defined and approved by the participants during the XIIIth European Workshop for Rheumatology Research held in Brighton, UK, January 28-30, 1993. Briefly, each participating centre was asked to enroll five patients with primary SS (group I), five with secondary SS (group II), five patients with well defined connective tissue diseases (CTD) but without SS (group III), and five control patients (group IV). The diagnosis of each CTD (for patients in group II and III) was established on the basis of well defined and commonly accepted criteria. ${ }^{2-6}$ The SS patients and disease controls enrolled in the present study should not have been included in the previous study aimed at defining the preliminary criteria set. 
The preliminary selection of patients with or without SS and their inclusion in one of the four disease groups was to be made on the basis of the investigator's clinical judgment and not on the basis of a single diagnostic parameter. There was no preliminary agreement regarding criteria or guidelines for the selection of SS patients; however, as all of the participants were experts in this particular disorder, it can be supposed that the patient selection criteria were fairly homogeneous across participating centres.

Control patients (group IV) were to be selected from those subjects referred to an SS expert because of ocular or oral signs and symptoms that simulated the clinical manifestations of SS, and for whom a complete evaluation was justified in order to establish a differential diagnosis.

This procedure for the preliminary selection of patients was the same as that adopted in part I of the study (the definition of the criteria ${ }^{7}$ ), and it was similar to the procedure used during the multicentre study by the American College of Rheumatology (ACR) (formerly the American Rheumatism Association (ARA)) for the revision of the classification criteria for rheumatoid arthritis (RA). ${ }^{2}$

Each subject was required to undergo the entire sequence of diagnostic procedures included in the classification criteria set (table 1), or at least a sufficient number of tests to confirm or exclude the diagnosis of SS. For instance, as the rules previously defined in the classification criteria set indicated that positive results in four of six tests was sufficient for a diagnosis of primary SS (table 1: rules for classification), in some subjects with a minimum of four positive items the remaining test(s) could be omitted. In the same way, some control subjects (group IV) could be defined on the basis of a minimum of three or six negative tests.

Table 1 Classification criteria for Sjögren's syndrome

I- Ocular symptoms: a positive response to at least one of the three selected questions: 1. Have you had daily, persistent, troublesome dry eyes for more than 3 months?

2. Do you have a recurrent sensation of sand or gravel in the eyes?

2. Do you have a recurrent sensation of sand or gravel in the
3. Do you use tear substitutes more than three times a day?

II- Oral symptoms: a positive response to at least one of the three selected questions:

1. Have you had a daily feeling of dry mouth for more than 3 months?

2. Have you had recurrently or persistently swollen salivary glands as an adult?

3. Do you frequently drink liquids to aid in swallowing dry food?

III-Ocular signs: objective evidence of ocular involvement defined as a positive result in at least one of the following two tests: ${ }^{13}$

1. Schirmer's I test $(\leqslant 5 \mathrm{~mm}$ in 5 minutes $) \S$

2. Rose Bengal score ( $\geqslant 4$ according to van Bijsterveld's scoring system)

IV-Histopathology: a focus score $\geqslant 1$ in a minor salivary gland biopsy.

(A focus is defined as an agglomerate of at least 50 mononuclear cells; the focus score is defined by the number of foci in $4 \mathrm{~mm}^{2}$ of glandular tissue)..$^{13}$

V- Salivary gland involvement: objective evidence of salivary gland involvement defined by a positive result in at least one of the following three diagnostic tests: ${ }^{13}$

1. Salivary scintigraphy

2. Parotid sialography

3. Unstimulated salivary flow ( $\leqslant 1.5 \mathrm{ml}$ in 15 minutes) $\$$

VI-Autoantibodies: presence in the serum of the following autoantibodies: ${ }^{13}$

1. Antibodies to Ro(SS-A) or $\mathrm{La}(\mathrm{SS}-\mathrm{B})$ antigens, or both

Rules for classification: In patients without any potentially associated disease the presence of any four of the six items is indicative of primary SS.

In patients with a potentially associated disease (for instance another connective tissue disease)

In patients with a potentially associated disease (for instance another connective tissue disea
item I or item II plus any two from among items III, IV, V is indicative of secondary SS.

item I or item II plus any two from among items III, IV, V is indicative of secondary SS.

Exclusion criteria: pre-existing lymphoma, acquired immunodeficiency disease (AIDS),
sarcoidosis, graft $v$ host disease, sialoadenosis. ${ }^{1{ }^{1}}{ }_{2}$ Use of antidepressant and anti-hypertensive sarcoidosis, graft $v$ host disease, sialoadenosis ${ }^{11}{ }_{12}$
drugs, neuloleptics, parasympatholytic drugs.

SAs it has been demonstrated that this test may be reduced in normal subjects older than 60 years, ${ }^{14}$ it should be excluded from the criteria or not considered indicative for a diagnosis of SS in elderly subjects.
Each diagnostic test was required to be performed following the directions previously defined. ${ }^{13} 14$ A standard clinical chart for recording the results was prepared and distributed to the participating centres.

\section{DATA ANALYSIS}

The completed clinical charts were sent by the participants back to the coordinating centre (University of Pisa), where they were collected and entered into a database developed from a standard program (Fox Base +/Mac, Fox Software) and run on an Apple MacIntosh II computer. To assess the ratios of correct classification, $2 \times 2$ tables were used (true positive/ false negative SS patients and true negative/ false positive non-SS controls). The rules for the classification (table 1) were derived from the preliminary criteria set and from the performance of different combinations of these criteria in part I of the study. ${ }^{7}$ However, it was decided not to include the differentiation between the 'probable' and 'definite' diagnosis. This was in agreement with the procedure followed by the Diagnostic and Therapeutic Criteria Committee of the ARA in revising the classification criteria for RA. ${ }^{2}$ Briefly, for primary SS the diagnosis was established by the presence of any four of the six items of the criteria set (item IV being limited to the presence of Ro(SS-A) or $\mathrm{La}(\mathrm{SS}-\mathrm{B})$ antibodies, or both), and in the absence of any disorder listed among the exclusion criteria or any disease potentially associated with SS (table 1). ${ }^{15}$ In patients with diseases potentially associated with sicca syndrome, the diagnosis of secondary SS was established when items I, II, or both, plus any two of items III-V were present (table 1). Because items $\mathrm{III}(\mathrm{a})$ and $\mathrm{V}(\mathrm{c})$ (Schirmer's I test and unstimulated whole saliva collection, respectively) had been found to be significantly reduced in the elderly control population of the previous study, ${ }^{14}$ it was decided to not consider these two tests in any individual older than 60 years (see footnote to table 1).

The classification tree or recursive partitioning procedure ${ }^{16}$ was also used as alternative method for the classification of SS patients and non-SS controls. Only those subjects (patients with primary SS and control patients without SS) for whom the entire six item criteria set was available were selected for this classification procedure. The sequence in the 'classification tree' is usually created by examining every allowable split of each variable for each node. The most discriminating split is that which creates two 'daughter' nodes of progressively higher purity-that is, nodes that contain progressively larger proportions of either SS patients only or non-SS controls.

Various statistical packages (Stat View II, Abacus Concepts Inc; Systat, Systat Inc) were used in performing the statistical procedures.

\section{Results}

Twenty four centres from 12 European countries, plus two centres from Israel returned 
Table 2 Main demographic features of the subjects included in the study, and distribution among the patients of the different connective tissue diseases (CTD) associated with SS (secondary SS) or not associated with SS (CTD no SS)

\begin{tabular}{|c|c|c|c|c|c|c|c|c|c|}
\hline Disease group & No & $\begin{array}{l}\text { Sex } \\
(M / F)\end{array}$ & $\begin{array}{l}\text { Age } \\
\text { (years) }\end{array}$ & $S L E$ & $R A$ & $S S c$ & $P M / D M$ & $M C T D$ & Others \\
\hline Primary SS (group I) & 81 & $2 / 79$ & $59 \cdot 0(46 \cdot 2$ to $66 \cdot 7)$ & - & - & - & - & - & - \\
\hline Secondary SS (group II) & 76 & $7 / 73$ & $55.0(45.5$ to 61.0$)$ & $17(22 \cdot 4)$ & $41(53.9)$ & $11(14 \cdot 5)$ & $0(0 \cdot 0)$ & $0(0 \cdot 0)$ & $7(9 \cdot 2)$ \\
\hline CTD no SS (group III) & 54 & $2 / 52$ & $47.0(39.0$ to 56.0$)$ & $19(35 \cdot 2)$ & $17(31 \cdot 5)$ & $7(13.0)$ & $1(1 \cdot 8)$ & $4(7 \cdot 4)$ & $6(11 \cdot 1)$ \\
\hline Controls (group IV) & 67 & $11 / 56$ & $50.5(38.0$ to 63.0$)$ & - & - & - & - & - & - \\
\hline
\end{tabular}

Values are number, median (interquartile range), or number (\%). SS = Sjögren's syndrome; SLE = systemic lupus erythematosus; $\mathrm{RA}=$ rheumatoid arthritis; $\mathrm{SSc}=$ systemic sclerosis; $\mathrm{PM} / \mathrm{DM}=$ polymyositis/dermatomyositis; $\mathrm{MCTD}=$ mixed connective tissue disease CTD = connective tissue disease.

data on SS patients and non-SS controls in a total of 388 cases. To reduce the possibility of selection bias, we took into account for the analysis of the results only data from centres that provided an adequate number of both SS patients and non-SS controls - that is, all or almost all of the patient cases and at least $70 \%$ of the control cases required by the study protocol. In this way we excluded the data from eight centres and limited the analysis to those provided by 16 centres from 10 countries, for a total number of 278 cases. It should be noted that four of the 16 centres did not take part in the previous study aimed at the definition of the criteria. ${ }^{7}$ Table 2 lists the main clinical and epidemiological characteristics of the patient populations.

The classification accuracy of the criteria set was first tested, as indicated in the previous study, ${ }^{7}$ considering the presence of any four of the six items (table 1) to be diagnostic for primary SS. The sensitivity of the criteria in correctly classifying primary SS patients was $97 \cdot 5 \%$ and its specificity in correctly classifying non-SS controls (patients from group III plus those from group IV) was $94 \cdot 2 \%$. The specificity was $92.5 \%$ when only group IV (disease controls without CTD) was taken as the control population (table 3 ). In classifying secondary SS, the sensitivity of the criteria set was $97.3 \%$ and the specificity was $91.8 \%$ when the presence of item I or II plus any two of items III-V was considered as indicative for such a diagnosis in patients with a CTD (table 3).

When the criteria set was simplified by excluding the tests for salivary gland involvement (item V)-which are considered to be invasive and expensive, and which are less commonly performed in clinical practice-this also reduced the accuracy of the criteria. When the presence of any four of the five remaining items was accepted as indicative of primary SS, the specificity was $98.3 \%$ ( 119 of 121 correctly classified controls) and the sensitivity was
$83 \cdot 7 \%$ (67 of 80 patients with primary SS correctly classified).

When patients and controls enrolled by the four centres that did not take part in the previous phase of the study were analysed separately, the results obtained were not significantly different from those derived from the analysis of the populations of SS patients and non-SS controls provided by the remaining 12 centres.

The figure shows the classification tree derived by applying the procedure of recursive partitioning to the patients with primary SS and the non-SS controls. The sequence of tests that gave the best split for each node was the same as that found in the first study. ${ }^{7}$ The overall performance of the classification tree was quite similar to the performance of the criteria set applied in no fixed sequence. The sensitivity was $95.6 \%$ (66 of 69 correctly classified patients with primary SS), and the specificity was $90.9 \%$ (70 of 77 correctly classified disease controls, of whom 30 were patients with CTD without SS).

A similar sensitivity and specificity was obtained when a four step sequence was used, in which tests for salivary gland involvement (item $\mathrm{V}$ of the criteria set) constituted the third step after the subjective reporting of symptoms of dry eye or dry mouth (first step) and ocular signs (second step), while histopathological investigation of the minor salivary glands was the fourth step. In this alternative classification tree, the serological tests for Ro(SS-A) and $\mathrm{La}(\mathrm{SS}-\mathrm{B})$ antibodies were excluded, as they did not further improve the overall performance of the recursive partitioning procedure.

We also compared the accuracy of our proposed classification criteria with that of previous criteria. ${ }^{8-12}$ In order to eliminate selection bias, we compared only those SS patients and disease controls in whom it was possible to apply all of the previously described criteria sets. In this comparison, all the earlier criteria sets showed a good specificity, but a

Table 3 Classification criteria for Sjögren's syndrome (SS): combined performance in classifying patients with primary or secondary SS and non-SS disease controls

\begin{tabular}{|c|c|c|c|c|}
\hline & $\begin{array}{l}\text { Sensitivity } \\
(\%)\end{array}$ & $\begin{array}{l}\text { No of cases } \\
(T P / T P+F N)\end{array}$ & $\begin{array}{l}\text { Specificity } \\
(\%)\end{array}$ & $\begin{array}{l}\text { No of cases } \\
(T N / T N+F P)\end{array}$ \\
\hline $\begin{array}{l}\text { Criteria set for the classification of primary SS } \\
\text { Any four of six items in table } 1 \\
\text { Primary SS } v \text { controls + CTD without SS } \\
\text { Primary SS } v \text { controls }\end{array}$ & $\begin{array}{l}97 \cdot 5 \\
97 \cdot 5\end{array}$ & $\begin{array}{l}(79 / 81) \\
(79 / 81)\end{array}$ & $\begin{array}{l}94 \cdot 2 \\
92 \cdot 5\end{array}$ & $\begin{array}{c}(114 / 121) \\
(62 / 67)\end{array}$ \\
\hline $\begin{array}{l}\text { Criteria set for the classification of secondary SS } \\
\text { Item I or item II plus any two of items III-V in table } 1 \\
\text { Secondary SS } v \text { CTD without SS }\end{array}$ & $97 \cdot 3$ & $(71 / 73)$ & $91 \cdot 8$ & $(45 / 49)$ \\
\hline
\end{tabular}

$\mathrm{TP}=$ True positive $\mathrm{FN}=$ false negative $\mathrm{TN}=$ true negative $\mathrm{FP}=$ false positive $; \mathrm{SS}=$ Sjögren's syndrome $\mathrm{CTD}=$ connective tissue disease. 


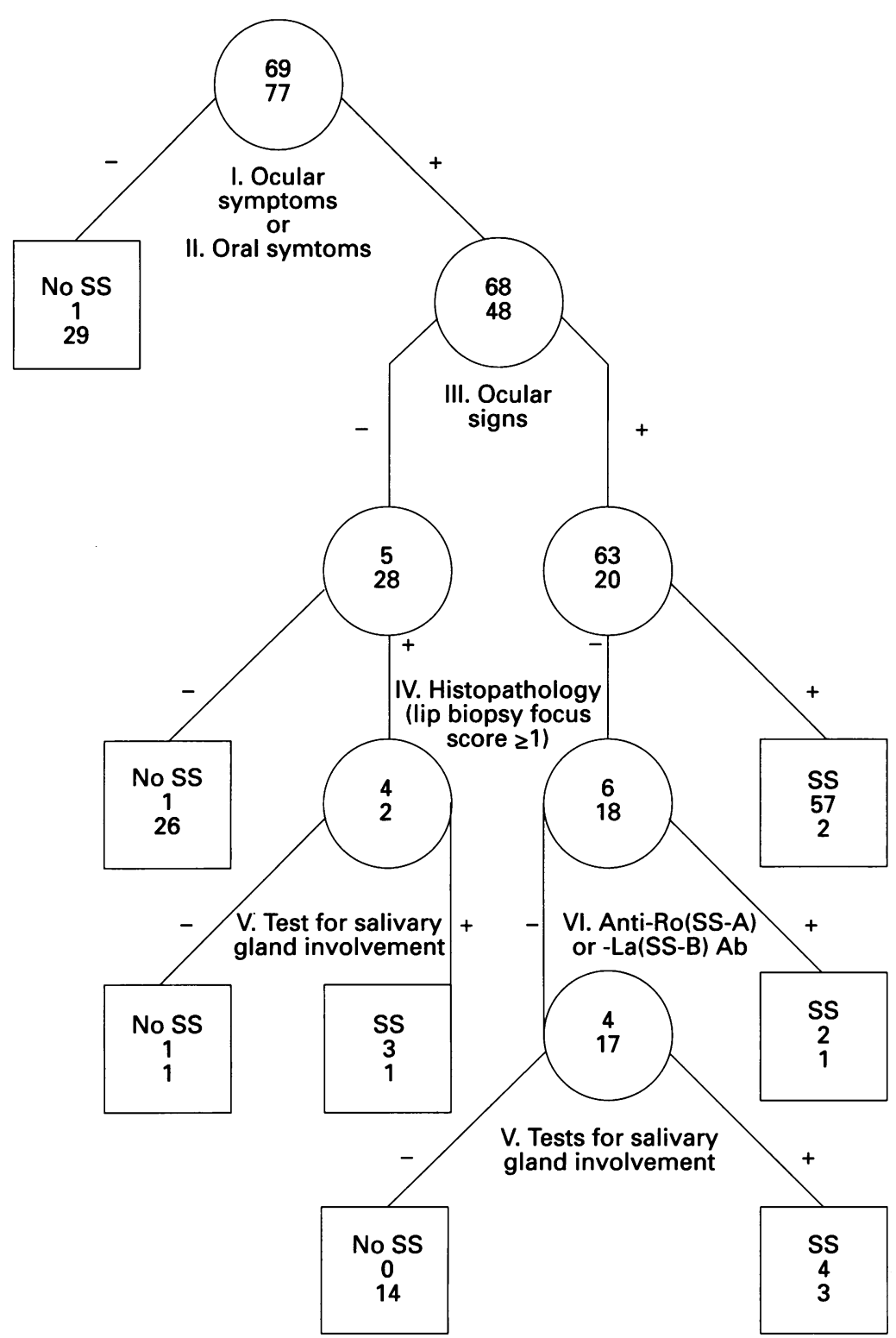

Schematic representation of the 'classification tree' for primary Sjögren's syndrome (SS). Within each circle appears the number of patients with primary SS (upper value) and the number of controls without SS (lower value). The boxes show the numbers of subjects who could be classified as either having SS or not having SS (No SS). The variable used in each node of the tree to discriminate between patients and controls is reported beneath the circles. The entire sequence allowed us to classify correctly 66 of the 69 patients with primary SS (sensitivity $95.5 \%$ ), and 70 of the 77 disease controls (specificity 90.9\%).

lower sensitivity and a significantly lower accuracy with respect to the European criteria set (table 4).

\section{Discussion}

In this study we evaluated the accuracy of a proposed set of classification criteria for SS defined by a multicentre European study. These criteria were tested in completely different populations of SS patients and nonSS disease controls recruited by 16 centres, some of which had not participated in the earlier definition of the criteria set.

The criteria showed an even better performance in terms of sensitivity and specificity than that observed in the preliminary study. This may be because slight modifications had been incorporated as a result of the significant number of false positive results observed in the measurement of tear and saliva production in the elderly population (Schirmer's I test and unstimulated whole saliva collection). This finding, based on a closer analysis of the results for the normal controls enrolled in the first phase of the study, ${ }^{14}$ confirmed reports in previous studies ${ }^{17} 18$ and induced us to define the criteria set more precisely when used in elderly patients; otherwise, four additional subjects older than 60 years would have been falsely classified as SS patients because of positivity of the Schirmer's I test, or the unstimulated whole saliva collection test, or both, and specificity would have decreased from $94 \cdot 2$ to $91 \cdot 7 \%$.

In contrast to the procedure followed earlier in defining the preliminary criteria for $\mathrm{SS},{ }^{7}$ we did not include in this criteria set the differentiation between 'definite' and 'probable' SS (for either the primary or the secondary variant of the syndrome). The principal aim of classification criteria is to improve specificity in the selection of patients for clinical studies, and therefore the concept of probability in disease classification could be considered as 'nonsense'. In keeping with this viewpoint, in their 1987 revision of the criteria for RA, the ARA Committee did not use the designations 'classic, definite, or probable' for the disorder, ${ }^{2}$ which had been included in the 1958 preliminary criteria. ${ }^{19}$

This European classification criteria set represents the first attempt to define criteria for SS using a statistical methodology that has already been used to define the classification criteria for other rheumatic diseases. ${ }^{2}$ Because a 'gold standard' is not available for most diseases, including SS, this methodology assumes that the clinician's judgment can be used as the 'gold standard' for both patients and controls. Of course, this methodology has some limitations. First, the individual judgment of different clinicians may result in some inhomogeneity of case selection. We can assume, however, that this bias is minimal

Table 4 Performance of previously suggested criteria for Sjögren's syndrome when used to classify patients with the primary syndrome and non-SS disease controlst

\begin{tabular}{lllllll}
\hline $\begin{array}{l}\text { Criteria set } \\
\text { (reference) }\end{array}$ & $\begin{array}{l}\text { Sensitivity } \\
(\%)\end{array}$ & $\begin{array}{l}\text { No of cases } \\
(T P / T P+F N)\end{array}$ & $\begin{array}{l}\text { Specificity } \\
(\%)\end{array}$ & $\begin{array}{l}\text { No of cases } \\
(T N / T N=F P)\end{array}$ & $\begin{array}{l}\text { Accuracy } \\
(\%)\end{array}$ & $\begin{array}{l}\text { No of cases } \\
(T P+T N / T P+F N+T N+F P)\end{array}$ \\
\hline Manthorpe et al ${ }^{8}$ & $71 \cdot 4$ & $25 / 35$ & $93 \cdot 5$ & $58 / 62$ & $85 \cdot 6^{\star \star \star}$ & $83 / 97$ \\
Skopouli et al & $57 \cdot 1$ & $20 / 35$ & $100 \cdot 0$ & $62 / 62$ & $84 \cdot 4^{\star \star \star}$ & $82 / 97$ \\
Homma et $^{10} l^{10}$ & $65 \cdot 7$ & $23 / 35$ & $100 \cdot 0$ & $62 / 62$ & $87 \cdot 6^{\star}$ & $85 / 97$ \\
Fox et al & $31 \cdot 4$ & $11 / 35$ & $100 \cdot 0$ & $62 / 62$ & $75 \cdot 2^{\star \star \star \star}$ & $73 / 97$ \\
Daniels et $a l^{12}$ & $40 \cdot 0$ & $14 / 35$ & $100 \cdot 0$ & $62 / 62$ & $69 \cdot 4^{\star \star \star \star}$ & $76 / 97$ \\
\hline
\end{tabular}

$\mathrm{TP}=$ True positive cases; $\mathrm{FN}=$ false negative cases; $\mathrm{TN}=$ true negative cases; $\mathrm{FP}=$ false positive cases.

Thirty five patients clinically classified as having primary SS and 62 classified as non-SS disease controls (26 patients from group III and 36 from group IV) in whom it was possible to apply both the European criteria and earlier criteria sets. . $^{8-12}$

${ }^{\star} \mathrm{p}<0.05,{ }^{\star \star \star} \mathrm{p}<0.005$ and ${ }_{\star \star \star \star} \mathrm{p}<0.0001$ (Fisher's exact test) with respect to the accuracy of the European criteria (96.9\%, ${ }_{\text {which }}<0.05, \star \star x p$
corresponds to 94 of 97 cases correctly classified). 
when clinicians expert in the specific field in question are involved. Second, the clinician's judgment is probably influenced by objective findings available at the moment of case recruitment. This implies a 'circular' bias that may artificially enhance the sensitivity and specificity of the derived criteria. Despite these limitations, however, this methodology is considered to be reliable, and was adopted by the ACR when they defined the classification criteria for RA and systemic lupus erythematosus. ${ }^{2}{ }^{3}$

A different approach to the problem might be first to define the disease using a preliminary classification set prepared by an expert committee, and then to assess how clinically defined cases fit this definition. This was the procedure adopted to define the earlier criteria for SS. ${ }^{8-12}$ However, as table 4 shows, the performance of at least some of the earlier criteria sets was inferior to that of the new European set.

In the present study, the fact that the complete sequence of tests was not carried out in $100 \%$ of the patients could have introduced an additional bias, particularly when the accuracy of the individual tests or of the different criteria sets were calculated and compared with each other. However, this potential source of statistical error was limited by the fact that the comparisons (between the accuracy of each diagnostic test alone and that of the European criteria set (table 5), and between the different classification criteria sets for SS (table 4)) were always carried out on the same populations of patients and controls for the two entities being compared (see footnotes to tables 4 and 5). It may also be pointed out that the purpose of the present study was not to assess the accuracy of the different diagnostic tests for oral and ocular involvement in SS (this was in fact the focus of an earlier study within the framework of this EC project ${ }^{14}$ ), but rather to demonstrate that the European criteria for the classification of SS represent a more valid and reliable instrument than those previously proposed for this purpose.

Using the European criteria set assessed in the present study, it is possible to establish a diagnosis of primary or secondary SS on the basis of a combination of subjective complaints, objective findings, and histopathological and serological data, independently of any single parameter. The advantages of this kind of approach are obvious. The accuracy of the combined application of a six item set is consistently greater than that of any single test, as shown in table 5. Among the individual tests, minor salivary gland biopsy was the most accurate for the diagnosis of SS (89.0 and 84.1 when focus scores $\geqslant 1$ and $>1$, respectively, were considered as indicative of the diagnosis), followed by parotid sialography $(83.3 \%)$, and Schirmer's I test $(77 \cdot 0 \%)$.

The present results do not confirm the hypothesis that the presence of focal lymphocytic sialoadenitis, defined by a focus score $>1$, is the 'sine qua non' criterion for the diagnosis of SS. ${ }^{122021}$ In the present series, the histopathological approach alone (when a focus score $>1$ is considered as diagnostic) showed a specificity similar to that obtained using a combination of four of the six items of the criteria set $(96 \cdot 1$ versus $93 \cdot 2 \%$ ), but its sensitivity was significantly lower $(68.3$ versus $97.5 \% ; \mathrm{p}<10^{-6}$ using Fisher's exact test) (data partially shown in table 5).

Finally, the possibility of using a combination of different tests allows one to classify correctly the whole population of patients suffering from SS-that is, those patients who complain of sicca symptoms and show ocular signs together with specific serological features. This subset of patients cannot be correctly classified and included in the heterogeneous population of SS patients when a more limited diagnostic approach is used. In contrast, these criteria could lead one to diagnose primary SS in patients lacking either focal sialoadenitis or specific autoantibodies, which are usually considered to be the most specific markers of the disease. In fact, the combination of positive results for items I, II, III and V, and negative results for IV and VI in the European classification set (table 1) was encountered only in six of the 81 patient cases (clinically defined as patients with primary SS), and in three disease controls (clinically defined as non-SS). When these were considered as misclassified, the accuracy of the criteria was slightly reduced $(94 \cdot 1$ rather than $95 \cdot 5 \%)$.

On the basis of the present assessment, it can be concluded that the criteria set recently proposed by the European Community study group is (with the inclusion of some slight modifications) highly valid and reliable in the classification of patients with either primary or secondary SS and could be adopted by the scientific community to standardise the diagnostic approach to this disorder and to

Table 5 Accuracy of the ocular and oral tests in the classification of patients with primary Sjögren's syndrome and disease controls compared with that of the European classification criteria

\begin{tabular}{|c|c|c|c|c|c|c|}
\hline & $\begin{array}{l}\text { No of } S S \\
\text { patients* }\end{array}$ & $\begin{array}{l}\text { Sensitivity (\%) } \\
(T P)\end{array}$ & $\begin{array}{l}\text { No of controls* } \\
\text { (groups III, IV) } \dagger\end{array}$ & $\begin{array}{l}\text { Specificity (\%) } \\
(T N) \text { (groups III, IV)† }\end{array}$ & $\begin{array}{l}\text { Accuracy (\%) } \\
(T P+T N / A l l)\end{array}$ & $\begin{array}{l}\text { Accuracy of the European criteria (\%) } \\
(T P+T N / \text { All }) \neq\end{array}$ \\
\hline Schirmer's I test & 80 & $86 \cdot 2(69)$ & $116(53-63)$ & $70 \cdot 7(82)(42-40)$ & $77 \cdot 0(151 / 196)$ & $95 \cdot 4(187 / 196)$ \\
\hline Rose Bengal score & 70 & $52.9(37)$ & $97(39-58)$ & $91.7(89)(35-54)$ & $75.4(126 / 167)$ & $94 \cdot 6(158 / 167)$ \\
\hline Focus score $\geqslant 1$ in MSGB & 79 & $87 \cdot 3(69)$ & $103(42-61)$ & $90 \cdot 3(93)(39-54)$ & $89.0(162 / 182)$ & $95 \cdot 1(173 / 182)$ \\
\hline Focus score $>1$ in MSGB & 79 & $68 \cdot 4(54)$ & $103(42-61)$ & $96 \cdot 1(99)(40-59)$ & $84 \cdot 1(153 / 182)$ & $95 \cdot 1(173 / 182)$ \\
\hline Salivary scintigraphy & 46 & $82 \cdot 6(38)$ & $59(26-33)$ & $62.7(37)(18-19)$ & $71 \cdot 4(75 / 105)$ & $95 \cdot 2(100 / 105)$ \\
\hline Parotid sialography & 29 & $72 \cdot 4(21)$ & $37(14-23)$ & $91.9(34)(12-22)$ & $83 \cdot 3(55 / 66)$ & $97 \cdot 0(64 / 66)$ \\
\hline Unstimulated saliva collection & 57 & $71.9(41)$ & $95(41-54)$ & $74 \cdot 7(71)(35-36)$ & $73 \cdot 7(112 / 152)$ & $94 \cdot 7(144 / 152)$ \\
\hline
\end{tabular}

^Numbers of patients with primary SS and control cases who underwent each test.

†Number of control cases from group III (patients with defined CTD without SS) and group IV (control patients)

†The accuracy of each test was compared with the accuracy of the European criteria set applied to the same group of SS patients and non-SS controls who performed that test.

TP = True positive cases; $T N=$ true negative cases, $\mathrm{All}=$ all patients with primary SS plus the control cases who performed each test; $M S G B=$ minor salivary gland biopsy. 
facilitate comparisons between patient populations from different centres. A definitive validation of these criteria is still needed, however, and should be performed in a large population of unselected individuals.

*Affiliations of the European Study Group on Diagnostic Criteria for Sjögren's Syndrome: Claudio Vitali, Marta Mosca, Maria Sciuto, Stefano Bombardieri: Clinical Immunology and Rheumatology Units, University of Pisa, Pisa, Italy; Haralampos $M$ Moutsopoulos: Department of Pathophysiology, Athens Medical School, Athens, Greece; Joaquin Coll: Department of Medicine, Hospital del Mar, Barcelona, Spain; Roberto Gerli: Institute of Internal Medicine and Oncological Sciences, University of Perugia, Perugia, Italy; Pierre Y Hatron: Service de Médecine Interne A, Hôpital Huriez, Lille, France; Louis Kater: Section of Clinical Immunology, University Hospital Utrecht, The Netherlands; Yriö T Konttinen: Institute of Biomedicine, Department of Yrjö T Konttinen: Institute of Biomedicine, Department of Anatomy, Helsinki, Finland; Rolf Manthorpe: Sjögren's Syndrome Research Centre, University Hospital, Malmö, Sweden; Olivier Meyer: Clinique Rhumatologique, Faculté
Xavier Bichat, Paris, France; Pierantonio Ostuni: Division of Xavier Bichat, Paris, France; Pierantonio Ostuni: Division of
Rheumatology, University of Padova, Padova, Italy; Raffaele A Rheumatology, University of Padova, Padova, Italy; Raffaele A
Pellerito: II Division of Medicine, Hospital Mauriziano, Torino, Italy; Yvon L Pennec: Department of Internal Medicine, Brest University Medical School Hospital, Brest, France; Stephen R Porter: Academic Department of Oral Medicine, Eastman Dental Hospital, London, UK; Andrea Richards: Department of Oral Medicine, Surgery and Pathology, Bristol Dental Hospital and School, Bristol, UK; Bernard Sauvezie: Clinical Immunology Unit, Hôpital Gabriel Mont Pied, Clermont Ferrand, France; Morten Schiødt: Hillerød Sjögren's Centre Ferrand, France; Morten Schiødt: Hillerød Sjögren's Centre and Department of Oral Medicine and Oral Surgery, Hillerød, Denmark; Yehuda Shoenfeld: Research Unit of Autoimmune Diseases, Sackler Faculty of Medicine, Tel Aviv, Israel; Fotini N Skopouli: Department of Internal Medicine, Ioannina, Greece; Josef S Smolen: 2nd Department of Medicine and Ludwig Boltzman Institute for Rheumatology, Vienna, Austria; Francisco Soremenho: Serviço Medicina 2, Hospital Curry Ichilov Medical Centre, Tel Aviv, Israel; Matija Tomsic: University Medical Centre, Department of Rheumatology, Ljubljana, Slovenia; Joop P van de Merwe: Department of Immunology, Erasmus University and University Hospital, Rotterdam, The Netherlands; Christine $M$ Yeoman: Depart-
ment of Oral and Maxillofacial Surgery, Royal Hallamshire Hospital, Sheffield, UK; Marie J Wattiaux: Hôpital Saint Antoine, Paris, France.

The following investigators (in alphabetical order by country)

also contributed in the performance of the study: J Hitzelhammer, P Petera: 2nd Department of Medicine and Ludwig Boltzman Institute for Rheumatology, Vienna, Austria; J Hietanen: Department of Oral Pathology, Helsinki, Finland; M Malmström: Department of Oral and Maxillofacial Surgery, Helsinki, Finland; P Youinou: Immunology Laboratory, Brest University Medical School Hospital, Brest, France; M Grossin Department of Pathology, Bichat Hospital, Paris, France; M F Kahn: Department of Rheumatology, Bichat Hospital, Medical Centre, Tel-Hashomer, Israel; A Ianniello, P Sfriso: Division of Rheumatology, University of Padova, Padova, Italy; R Ercolani, C Muscat: Institute of Internal Medicine and Oncological Sciences, University of Perugia, Perugia, Italy; R De Giovanni, I Rizzi: II Division of Medicine, Hospital R De Giovanni, L Rizzi: II Division of Medicine, Hospital Mauriziano, Torino, Italy; F H J van den Hoogen: Department of Rheumatology, University Hospital, Nijmegen, The Netherlands; H Hoodkaas: Department of Immunology, Erasmus University and University Hospital, Rotterdam, The
Netherlands; A A Kruize: Department of Rheumatology, University Hospital, Utrecht, The Netherlands; J Alves: Serviço Medicina 2, Hospital Curry Cabral, Lisbon, Portugal; M Presetnik, B Rozman: University Medical Centre, Department of Rheumatology, Ljubljana, Slovenia; T Axell, Å Larsson H Tabety: Sjögren's Syndrome Research Centre, University Hospital, Malmö, Sweden; P J Maddison: Royal National Hospital for Rheumatic Diseases, Bath, UK. S Mutlu: University of Bristol Dental Hospital, Bristol, UK; C Scully:
Joint Department of Oral Medicine, Eastman Dental Institute, London, UK; C D Franklin: Department of Oral Pathology, University of Sheffield, Sheffield, UK; M Nelson: Department of Ophthalmology, Royal Hallamshire Hospital, Sheffield, UK.

1 Fries J F, Hochberg M C, Medsger T A, Hunder G G, Bombardier C, and the American College of Rheumatology Diagnostic and Therapeutic Criteria Committee. Criteria for rheumatic diseases. Different types and functions. Arthritis Rheum 1994; 37: 454-62.

2 Amett F C, Edworthy S M, Bloch D A, et al. The American Rheumatism Association 1987 revised criteria for the classification of rheumatoid arthritis. Arthritis Rheum 1988; 31: 315-24.

3 Tan E M, Cohen A S, Fries J F, et al. The 1982 revised criteria for the classification of systemic lupus erythematosus. Arthritis Rheum 1982; 25: 1271-7.

4 Masi A T, Rodnan G P, Medsger T A, et al. Preliminary criteria for the classification of systemic sclerosis (scleroderma). Arthritis Rheum 1980; 23: 581-90.

5 Sharp G C, Irvin W S, Tan E M, Gould R G, Holman H R. Mixed connective tissue disease. An apparently distinct rheumatic disease syndrome associated with a distinct rheumatic disease syndrome associated with a (ENA). Am $\mathcal{F}$ Med 1972; 52: 148-59.

6 Bohan A, Peter J B. Polymyositis and dermatomyositis. Part I and Part II. N Engl F Med 1975; 292: 344, 403.

7 Vitali C, Bombardieri S, Moutsopoulos H M, et al. Preliminary criteria for the classification of Sjögren's syndrome. Results of an EEC prospective concerted action. Arthritis Rheum 1993; 36: 340-7.

8 Manthorpe R, Oxholm P, Prause J U, Schiödt M. The Copenhagen criteria for Sjögren's syndrome. Scand $f$ Rheumatol 1986; (suppl 61): 19-21.

9 Skopouli F N, Drosos A A, Papaioannou T, Moutsopoulos H M. Preliminary diagnostic criteria for Sjögren's syndrome. Scand $\mathcal{F}$ Rheumatol 1986; (suppl 61): 22-5.

10 Homma M, Tojo T, Akizuki M, Yagamata H. Criteria for Sjögren's syndrome in Japan. Scand $\mathcal{F}$ Rheumatol 1986; (suppl 61): 26-7.

11 Fox R I, Robinson C, Curd J C, Michelson P, Kozin F, Howell F V. Siögren's syndrome: proposed criteria for classification. Arthritis Rheum 1986; 29: 577-85.

12 Daniels T E, Talal N. Diagnosis and differential diagnosis of Sjögren's syndrome. In: Talal N, Moutsopoulos H M, Kassan S S, eds. Sjögren's syndrome: clinical and immunological aspects. Berlin: Springer-Verlag, 1987; 193-9.

13 Workshop on Diagnostic Criteria for Sjögren's syndrome. I. Questionnaires for dry eye and dry mouth. II. Manual of methods

14 Vitali C, Moutsopoulos H M, Bombardieri S, and The European Community Study Group on Diagnostic European Community Study Group on Diagnostic Criteria for Sjögren's syndrome. The European Community Study Group on Diagnostic Criteria for
Sjögren's syndrome. Sensitivity and specificity of tests for Sjögren's syndrome. Sensitivity and specificity of tests for
ocular and oral involvement in Siögren's syndrome. Ann ocular and oral involvement in
Rheum Dis 1994; 53: 637-47.

5 Moutsopoulos H M, Chused T M, Mann D L, et al. Sjögren's syndrome (sicca syndrome): current issues. Ann Intern Med 1980; 92: 212-26.

16 Breiman L, Friedman J H, Olshen R A, Stone C J. Classification and regression trees. Belmont, CA: Wadsworth, 1985.

17 Seal D V. The effect of ageing and disease on tear constituents. Trans Ophthalmol Soc UK 1985; 104: 355-62.

18 Heft M W, Baum B J. Unstimulated and stimulated parotid flow rate in individuals of different ages. $\mathcal{F}$ Dent Res 1984 ; 63: $1182-5$.

19 Ropes M W, Bennett G A, Cobb S, Jacox R, Jessar R A. 1958 revision of diagnostic criteria for rheumatoid arthritis. Bull Rheum Dis 1958; 9: 175-6.

20 Daniels T E, Whitcher J P. Association of patterns of labial salivary gland inflammation with keratoconjunctivitis sicca. Analysis of 618 patients with suspected Sjögren's syndrome. Arthritis Rheum 1994; 37: 869-77.

21 Daniels T E. Labial salivary gland biopsy in Siögren's syndrome: assessment of a diagnostic criterion in 362 suspected cases. Arthritis Rheum 1984; 27: 147-56. 